\title{
Repetitive Control for a Class of Nonlinear Systems*
}

\author{
Hiromitsu HIKITA**, Mitsuhisa YAMASHITA** \\ and Yuzuru KUBOTA**
}

\begin{abstract}
A repetitive control scheme which is applicable to a class of nonlinear systems with periodic reference signals is proposed. The nonlinear terms in the systems are assumed to be polynomially bounded. Sliding mode control is applied to an augmented system in which an integrator and a repetitive controller are combined in order to obtain a linear input-output relation. The stability condition for the repetitive control system realized by the proposed method is derived. It is shown that this system satisfies the internal model principle for a given frequency bandwidth. The frequency bandwidth is given by the stability condition. An illustrative example is given.
\end{abstract}

Key Words: Automatic Control, Robotics, Repetitive Control, Sliding Mode Control, Learning Control

\section{Introduction}

There are many systems which are required to repeat the same motion in each of a series of periods of constant length. Learning control ${ }^{(1),(2)}$, iterative control $^{(3)}$ and repetitive control ${ }^{(4)-(8)}$ are effective to control such systems. In order to reduce the tracking error for every period in control systems realized by these control methods, not only the tracking error in the present period but also those in previous periods are used. Therefore, these control methods are evidently different from the method of a typical servomechanism whose control input depends on only the present tracking error. The structures of these control systems can be considered to be basically similar. The output is reset at the beginning of every period in a learning and an iterative control system. However, the reset operation is not required in a repetitive control system. The best control method will be chosen from the viewpoint of suitability for practical

* Received 7th August, 1992. Paper No. 90-1389 B

** Muroran Institute of Technology, 27-1 Mizumotocho, Muroran, Hokkaido 050, Japan problems. In this paper, repetitive control is investigated in order to extend the application field.

The repetitive control is based on the internal model principle ${ }^{(9)}$, which plays an important role when we construct linear servomechanisms. Zero tracking error can be achieved in the steady state by introducing the model of a periodic reference-signal generator in the closed loop. Therefore, many useful results have been derived in the linear repetitive control theory to date. However, there is no study on nonlinear repetitive control systems, except for one in which the stability of the total system is discussed. In the research, the same type of controller as the one built for a linear repetitive control system is used. In this paper, a design method is proposed which satisfies the internal model principle by application of sliding mode control ${ }^{(11)-(14)}$ to a nonlinear system in which the nonlinear terms are polynomially bounded. In addition, the control method is applied to the repetitive motion of a two-link manipulator, and the tracking property is investigated by means of simulation.

\section{Basic Principle}

Consider a nonlinear, time-varying system de- 
scribed by

$$
\begin{aligned}
& y_{j}^{\left(n_{j}\right)}=f_{j}\left(Y_{1}, Y_{2}, \cdots, Y_{m}, t\right)+b_{j}(t) u_{j}+d_{j}(t), \\
& \quad j=1,2, \cdots, m
\end{aligned}
$$

where $u_{j}$ and $y_{j}, j=1,2, \cdots, m$ are the input and the output. The term $d_{j}(t)$ represents the disturbance. $Y_{j}$ is

$$
Y_{j}=\left[y_{j} \dot{y}_{j} \cdots y_{j}^{\left(n_{j}-1\right)}\right]^{T}, \quad j=1,2, \cdots, m
$$

It is assumed that $f_{j}$ is a nonlinear function which is polynomially bounded, as explained later, and that the varying ranges of $b_{j}(t)$ and $d_{j}(t), j=1,2, \cdots, m$ are known. It is also assumed that $b_{j}(t)>0, j=1,2, \cdots, m$ without loss of generality.

With the introduction of state variables, system (1) can be described as

$$
\begin{aligned}
& \dot{x}_{j i}=x_{j, i+1}, j=1,2, \cdots, n_{j}-1 \\
& \dot{x}_{j, n_{j}}=f_{j}\left(X_{1}, X_{2}, \cdots, X_{m}, t\right)+b_{j}(t) u_{j}+d_{j}(t), \\
& j=1,2, \cdots, m \\
& X_{j}=\left[x_{j 1} x_{j 2} \cdots x_{j n_{j}}\right]^{T}
\end{aligned}
$$

where $y_{j}=x_{j 1}, j=1,2, \cdots, m$.

Let the period of the periodic reference signal $r_{j}$, $j=1,2, \cdots, m$ be $L$. Therefore,

$$
r_{j}(t)=r_{j}(t-L), t<L, j=1,2, \cdots, m
$$

Consider the augmented system shown in Fig. 1, which is constructed by connecting a linear dynamic compensator to system (1). Term $e^{-L s}$ represents a time delay element with delay time $L$ and $q$ is a filter which will be investigated later. We assume that $L$ is the same for each $j$, as is $q$, since we can easily extend the obtained results to the cases of different values of $L$ and $q$ for each $j$. In order to apply the sliding mode control to this augmented system, switching hyperplanes $\sigma_{j}=0, j=1,2, \cdots, m$ are introduced, where

$$
\begin{aligned}
\sigma_{j} & =c_{j o} z_{j}+\sum_{i=1}^{n_{j}} c_{j i} x_{j i}, \quad c_{j, n_{j}}=1, \\
& j=1,2, \cdots, m
\end{aligned}
$$

While $\sigma_{j}=0$ holds by the switching feedback rule which is presented in the next section, the following relation is derived from Eqs. (2) and (4) and Fig. 1.

$$
Y_{j}(s)=\frac{\left.\left\{1+G_{L}(s)\right\} G_{j}(s)\right\}}{1+G_{L}(s) G_{j}(s)} R_{j}(s)
$$

where $Y_{j}(s)$ and $R_{j}(s)$ are the Laplace transforms of $y_{j}$ and $r_{j}$ and

$$
G_{\dot{L}}(s)=\frac{q e^{-L s}}{1-q e^{-L s}}
$$

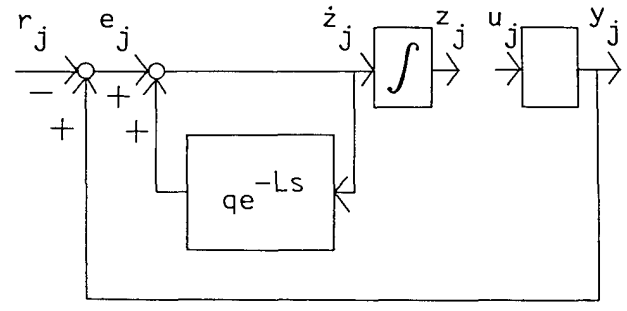

Fig. 1 Augmented system

$$
G_{j}(s)=c_{j o} / \sum_{i=0}^{n_{j}} c_{j i} s_{i}
$$

Therefore, $E_{j}(s)=\mathscr{L}\left\{e_{j}(t)\right\}$ is

$$
E_{j}(s)=\frac{G_{j}(s)-1}{1+G_{L}(s) G_{j}(s)} R_{j}(s)
$$

Consequently, from Eqs. ( 7 ) and ( 8 )

$$
\begin{aligned}
& E_{j}(s)=q e^{-L s}\left\{1-G_{j}(s)\right\} E_{j}(s) \\
& \quad+\left(1+q e^{-L s}\right)\left\{1-G_{j}(s)\right\} R_{j}(s)
\end{aligned}
$$

Figure 2 represents relation ( 9$)$. Then, the following result is derived from the small-gain theorem. If

i ) $G_{j}(s)$ is stable and

ii ) $\left|q\left\{1-G_{j}(s)\right\}\right|<1$

the tracking error $e_{j}(t)$ is bounded for a continuous and bounded $r_{j}(t)$.

If we choose the coefficients of Eq. (4) so that a wide frequency bandwidth is realized, a good convergence property of repetitive control will be given. Furthermore, since $G_{j}(s)$ in Eq. ( 7$)$ is the input-output relation of the first period, the response is improved in the first period. However, a suitable tradeoff is necessary since we cannot obtain extremely quick motion.

\section{Sliding Mode Control}

The input-output relation has been discussed in the previous section under the assumption that $\sigma_{j}=0$, $j=1,2, \cdots, m$, i.e., the sliding mode holds. In this chapter, we explain the control algorithm which satisfies the assumption.

Since $f_{j}$ is polynomially bounded, there are polynomials $F_{j}(X, t), j=1,2, \cdots, m$ such that

$$
\left|f_{j}(X, t)\right| \leqq F_{j}(|X|, t), j=1,2, \cdots, m
$$
where

$$
\begin{aligned}
& X=\left[X_{1}^{T} X_{2}^{T} \cdots X_{m}^{T}\right]^{T} \\
& |X|=\left[\left|X_{1}\right|^{T}\left|X_{2}^{2}\right|^{T} \cdots\left|X_{m}\right|^{T}\right]^{T} \\
& \left|X_{j}\right|=\left[\left|x_{j 1}\right|\left|x_{j 2}\right| \cdots\left|x_{j n_{j}}\right|\right]^{T}, \\
& \quad j=1,2, \cdots, m
\end{aligned}
$$

The coefficients of $F_{j}(X, t)$ can always be chosen to be continuously differentiable and positive functions of time without loss of generality. Therefore, the $k$-th term of $F_{j}(X, t)$ can be described as

$$
\eta_{j, k_{j}} \prod_{i=1}^{m} \prod_{p=1}^{n_{i}}\left(x_{i p}\right)^{\nu\left(j, i, p, k_{j}\right)}
$$

where $\eta_{j, k_{j}}$ is a positive coefficient and $\nu\left(j, i, p, k_{j}\right)$

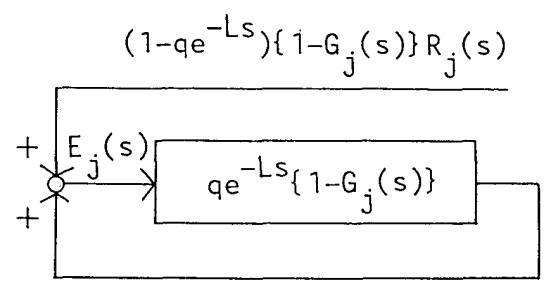

Fig. 2 System described by Eq. (9) 
represents the power.

With differentiation of Eq. (4) by time $t$,

$$
\dot{\sigma}_{j}=c_{j o} \dot{z}_{j}+c_{j 1} x_{j 2}+\cdots+c_{j, n j-1} x_{j, n_{j}}
$$$$
+f_{j}(X, t)+b_{j} u_{j}+d_{j}
$$

Let input $u_{j}$ be

$$
u_{j}=\phi_{j o} \dot{z}_{j}+\sum_{i=2}^{n_{j}} \phi_{j i} x_{j i}+\sum_{k_{j}} v_{j, k_{j}}(X, t)+\phi_{j}
$$

where $\phi_{j o}, \phi_{j i}, i=2,3, \cdots, n_{j}$ and $\phi_{j}, j=1,2, \cdots, m$ are switched coefficients. The rule of switching is derived later. Nonlinear terms $v_{j, k_{j}}(X, t), j=1,2, \cdots, m$ are given by

$$
v_{j, k_{j}}(X, t)=\xi_{j, k_{j}}(X, t) \prod_{i=1}^{m} \sum_{p=1}^{n_{i}}\left(x_{i p}\right)^{\nu\left(j, i, p, k_{j}\right)}
$$

where $\xi_{j, k_{j}}$ is a switched coefficient. Then, since

$$
\begin{aligned}
& \sigma_{j} \dot{\sigma}_{j}=\left(b_{j} \psi_{j o}+c_{j o}\right) \dot{z}_{j} \sigma_{j}+\left(b_{j} \psi_{j 2}+c_{j 1}\right) x_{j 2} \sigma_{j} \\
& \quad+\cdots+\left(b_{j} \psi_{j, n_{j}}+c_{j, n_{j}-1}\right) x_{j, n_{j}} \sigma_{j} \\
& \quad+\left\{b_{j} \sum_{k_{j}} v_{j, k_{j}}(X, t)+f_{j}(X, t)\right\} \sigma_{j} \\
& \quad+\left(b_{j} \phi_{j}+d_{j}\right) \sigma_{j}, j=1,2, \cdots, m
\end{aligned}
$$

$d \sigma_{j}^{2} / d t=2 \sigma_{j} \dot{\sigma}_{j}<0\left(\sigma_{j} \neq 0\right)$ is guaranteed, and the trajectory stays in the hyperplane $\sigma_{j}=0$ in the state space by switching $\psi_{j o}, \psi_{j i}, i=2,3, \cdots, n_{j}, \xi_{j, k_{j}}$ and $\phi_{j}$ as follows :

$$
\begin{aligned}
& \psi_{j o} \leqq \min _{b_{j}}\left(-c_{j o} / b_{j}\right) \text { if } \dot{z}_{j} \sigma_{j}>0 \\
& \psi_{j o} \geqq \max _{b_{j}}\left(-c_{j o} / b_{j}\right) \text { if } \dot{z}_{j} \sigma_{j}<0 \\
& \psi_{j i} \leqq \min _{b_{j}}\left(-c_{j, i-1} / b_{j}\right) \text { if } x_{j i} \sigma_{j}>0 \\
& \phi_{j i} \leqq \max _{b_{j}}\left(-c_{j, i-1} / b_{j}\right) \text { if } x_{j i} \sigma_{j}<0, \\
& \quad i=2,3, \cdots, n_{j} \\
& \xi_{j, k_{j}} \leqq-\frac{\eta_{j, k_{j}}}{b_{\min }} \text { if } \prod_{i=1}^{m} \prod_{p=1}^{n_{i}}\left(x_{i p}\right)^{\nu\left(j, i, p, k_{j}\right)} \sigma_{j}>0 \\
& \xi_{j, k_{j}} \geqq-\frac{\eta_{j, k_{j}}}{b_{\max }} \text { if } \prod_{i=1}^{m} \prod_{p=1}^{n_{i}}\left(x_{i p}\right)^{\nu\left(j, i, p, k_{j}\right)} \sigma_{j}<0 \\
& \phi_{j}=-k_{j} \operatorname{sgn} \sigma_{j}, k_{j}>\max _{d_{j, b}}\left|d_{i}\right| / b_{j}
\end{aligned}
$$

\section{Design Procedure and Properties of the Control System}

In order to obtain an effective repetitive control system, Eq. $(10 \cdot a)$ must be satisfied. This requirement can always be realized by designing a suitable switching hyperplane, as we can easily understand from Eqs. (4) and (7).

The design procedure can be summarized as follows.

(1) We construct an augmented system as Fig.1. Then, we determine the values of switching hyperplane (4). We choose the coefficients so that transfer function $(7)$ is stable.

(2) We design a filter $q$ which satisfies Eq. (10・b).

(3) We then switch the coefficients of control input (15) so that Eqs. (18) and (19) are always valid.

We now investigate whether or not the repetitive control system derived in this paper satisfies the internal model principle. A periodic reference signal with period can be considered to be an output signal of the system described in Fig.3. The transfer function of this system is $1 /\left(1-e^{-L s}\right)$. The loop transfer function of the repetitive control system in which the sliding mode holds is given by

$$
\frac{G_{j}(s)}{\left(1-q e^{-L s}\right)\left(1-G_{j}(s)\right)}
$$

If $q=1$, the loop transfer function includes the same transfer function as the reference signal generator. This means that the repetitive control system satisfies the internal model principle. Since the filter $q$ must be a low-pass filter in general, the internal model principle, exactly speaking, does not hold in this system. However, we can say that the principle holds for a restricted frequency bandwidth. This is the same restriction as in the case of repetitive control which is applied to a linear system with no direct path between input and output.

Since the above discussion is valid while $\sigma_{j}=0$ holds, it is desirable that the initial values are set such that $\sigma_{j}=0, j=1,2, \cdots, m$ at the beginning of control. Although $\sigma_{j}=0, j=1,2, \cdots, m$ are not attained at the beginning, they are automatically attained later and then the repetitive control works effectively since always $\sigma_{j} \dot{\sigma}_{j}<0 \quad\left(\sigma_{j} \neq 0\right), j=1,2, \cdots, m$ by the switching control algorithm.

\section{Application to a Two-Link Manipulator}

Consider the two-link manipulator in Fig. 4. Both the length and the mass of each link will be assumed normalized to unity. Therefore, there is no unit for length in figures in this paper. We define $U_{1}$ and $U_{2}$ as

$$
\begin{aligned}
& u_{1}=(4 / 3) T_{1}-\left(4 / 3+2 \cos \theta_{2}\right) T_{2} \\
& u_{2}=-\left(4 / 3+2 \cos \theta_{2}\right) T_{1}+\left(20 / 3+4 \cos \theta_{2}\right) T_{2}
\end{aligned}
$$

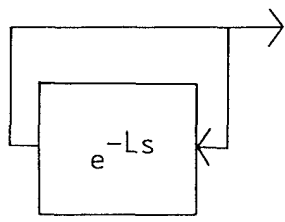

Fig. 3 Reference signal generator

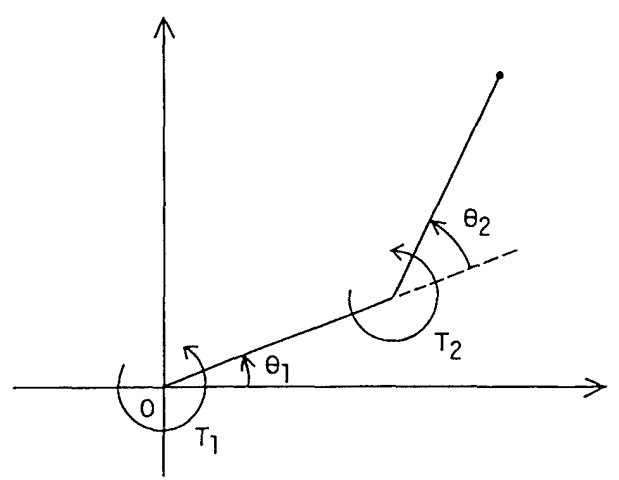

Fig. 4 Two-link manipulator 


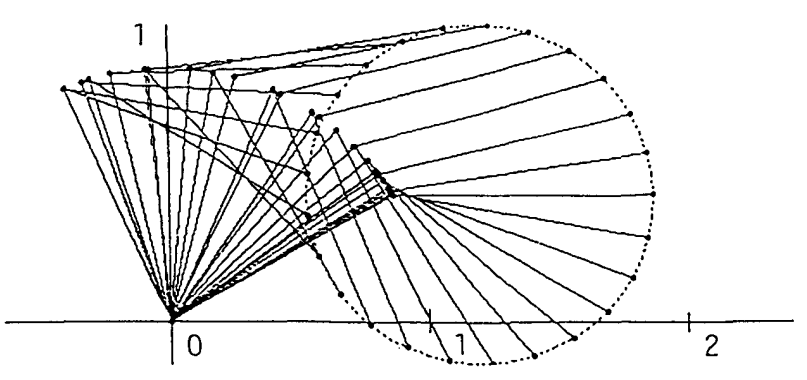

Fig. 5 Stick figures of the manipulator

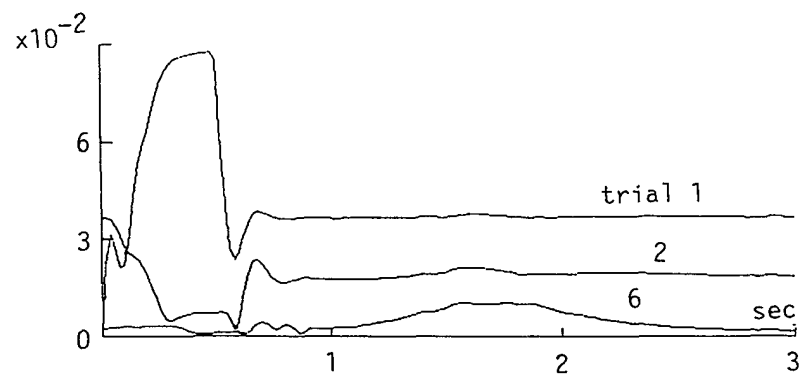

Fig. 6 Distance error of the manipulator hand

Let $x_{11}=\theta_{1}-\theta_{10}, x_{12}=\dot{\theta}_{1}, x_{21}=\theta_{2}-\theta_{20}$ and $x_{22}=\dot{\theta}_{2}$. The following state-space description is derived ${ }^{(12)}$.

$$
\begin{aligned}
\dot{x}_{11} & =x_{12} \\
\dot{x}_{12} & =\frac{1}{16 / 9-\cos ^{2} x_{21}}\left\{\frac{2}{3}\left(\sin x_{21}\right) x_{22}\left(2 x_{12}+x_{22}\right)\right. \\
& \left.+\left(\frac{2}{3}+\cos x_{21}\right)\left(\cos x_{21}\right) x_{12}^{2}+u_{1}\right\} \\
\dot{x}_{21} & =x_{22} \\
\dot{x}_{22} & =\frac{1}{16 / 9-\cos ^{2} x_{21}}\left\{-\left(\frac{2}{3}+\cos x_{21}\right)\left(\sin x_{21}\right) x_{22}\right. \\
& \left.\times\left(2 x_{12}+x_{22}\right)-2\left(\frac{5}{3}+\cos x_{21}\right)\left(\sin x_{21}\right) x_{12}^{2} u_{2}\right\} \\
y_{1} & =x_{11} \\
y_{2} & =x_{21}
\end{aligned}
$$

where $\theta_{10}$ and $\theta_{20}$, which are assumed to be zero, are $\theta_{1}$ and $\theta_{2}$ at the idling position of the manipulator, respectively.

We assume that the hand of the manipulator is required to circulate counter-clockwise at a fixed speed along the closed trajectory indicated in Fig.5. The pattern is a circle. The repetitive period is $3 \mathrm{sec}$. The initial values are $x_{11}=\pi / 6 \mathrm{rad}, x_{21}=-\pi / 6 \mathrm{rad}$ and $x_{12}=x_{22}=0$. This means that the hand of the manipulator starts from the right side of the reference circle with zero initial velocity. The initial value of $z_{i}$ is zero. We have chosen $\sigma_{i}=1200 z_{i}+70 x_{i 1}+x_{i 2}, \quad i=1,2$. Obviously, $\sigma_{j}=0, i=1,2$ are not attained at the starting point. A first-order lag system $1 /(0.03 s+1)$ has been chosen as the filter $q$ in order to satisfy the stability condition for this $\sigma_{i}$.

The switching control algorithm can be easily derived by application of the theoretical results in this
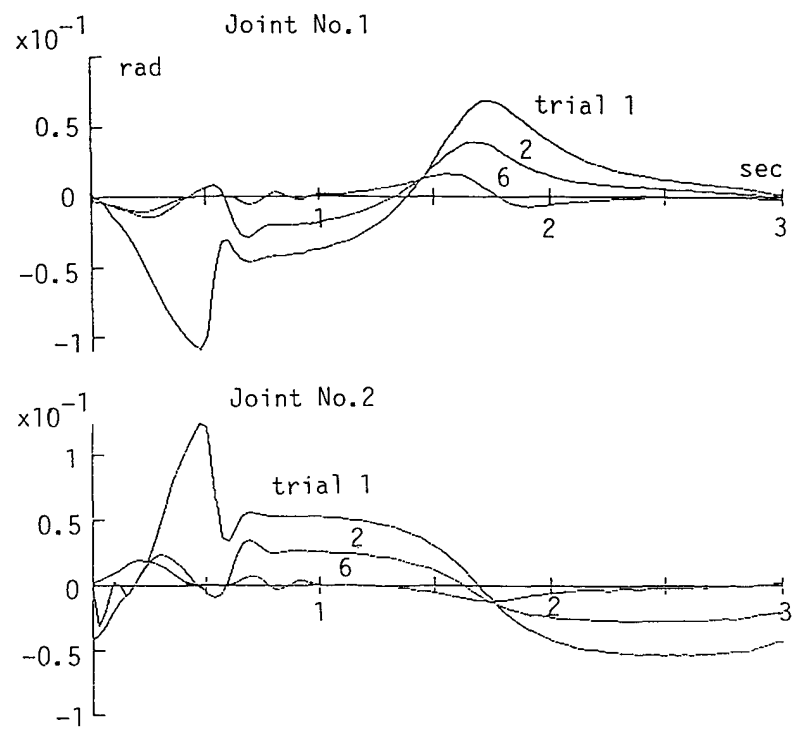

Fig. 7 Angular error of the manipulator arm

paper. The coefficients of nonlinear terms $x_{12} x_{22}, x_{22}^{2}$ and $x_{12}^{2}$ in $\dot{x}_{i 2}, i=1,2$ of the state equation of the manipulator are functions of sine and cosine which vary from -1 to 1 . Therefore, the maximum varying ranges of the coefficients can be calculated. Nonlinear terms $x_{12} x_{22}, x_{22}^{2}$ and $x_{12}^{2}$ and $\dot{z}_{i}$ and $x_{i 2}$ have been fed back as $u_{i}$ and the coefficients of these terms have been switched so that $\sigma_{i} \dot{\sigma}_{i}<0 \quad\left(\sigma_{i} \neq 0\right)$ holds. The minute details will be omitted.

Figure 6 shows the distance error between the reference and actual positions of the hand. Figure 7 shows the angular error between the reference and actual angles of the arm at each joint. The results of trials 1, 2 and 6 are given in Figs. 6 and 7. It is found that the tracking error decreases with every trial.

The sampling period was $1 \mathrm{msec}$ in this simulation. Serious chattering may occur in practical operation if a long sampling period is adopted. It is necessary to use a short sampling period by means of a high -speed signal processor such as a DSP. A certain amount of chattering does not affect the convergence property of repetitive control. However, it will increase the final tracking error.

\section{Conclusions}

A design method of a repetitive control system using sliding mode control has been proposed for a nonlinear system. The derived system satisfies the internal model principle for a frequency bandwidth which is determined by the stability condition. Although the sliding mode control investigated in this paper is different from typical sliding mode control, which is usually used to maintain an equilibrium point, the robustness property for disturbances and parameter variations is preserved. Since control inputs are 
given by high-speed switching, chattering occurs in general. However, since many physical systems have the property of a low-pass filter, and the chattering is reduced in output signals, the convergence process of the repetitive control is almost unaffected. In order to avoid chattering, it is possible to apply quasi-sliding mode control ${ }^{(12),(15)}$ in which the amplitude of the switching control is continuously interpolated.

\section{References}

(1) Kawamura, S., Miyazaki, F. and Arimoto, S., System Theoretic Study on Learning Control Method, Trans. Soc. Instr. Contr., (in Japanese), Vol. 21, No. 5 (1985), p. 445.

(2) Kawamura, S., Miyazaki, F. and Arimoto, S., Proposal of Betterment Process: A Learning Control Method for Dynamical Systems, Trans. Soc. Instr. Contr., (in Japanese), Vol. 22, No. 1 (1986), p. 56.

(3) Mita, T., Kato, E. and Aoki, Y., Iterative Control and Its Applications to Trajectory Control of Robot Manipulators, Trans. Soc. Instr. Contr., (in Japanese), Vol. 21, No. 11 (1985), p. 1223.

(4) Inoue, T., Iwai, S. and Nakano, M., High Accuracy Control of Play-Back Servo Systems, Trans. IEE of Japan, (in Japanese), Vol. 100, No. 7, C (1980), p. 89.

(5) Inoue, T., Nakano, M., Kubo, T., Matsumoto, S. and Baba, H., High Accuracy Control of a Proton Synchrotron Magnet Power Supply, Preprint 8th IFAC World Congr., Vol. 10 (1981), p. 216.

(6) Hara, S., Omata, T. and Nakano, M., Stability Condition and Synthesis Methods for Repetitive
Control Systems, Trans. Soc. Instr. Contr., (in Japanese), Vol. 22, No. 1 (1986), p. 36.

( 7 ) Hikita, H. Yamashita, M. and Kubota, Y., ModelFollowing Repetitive Control, Trans. Jpn. Soc. Mech. Eng., (in Japanese), Vol.55, No. 519, C (1989), p. 2792.

(8) Hikita, H. and Kyoutoku, S., A Repetitive Control System of a Model Following Type, Proc. 28th IEEE CDC, Vol. 1 (1989), p. 757.

(9) Soc. Instr. Contr. Engineers ed., Control Engineering Handbook, (in Japanese), (1983), p.757, Ohm-sha.

(10) Nakano, M., Inoue, T., Yamamoto, Y. and Hara, S., Repetitive Control, (in Japanese), (1989), p. 129, Soc. Instr. Contr. Engineers.

(11) Utkin, V.I., Variable Structure System with Sliding Mode, Trans. IEEE, Vol. AC-22, No. 2 (1979), p. 212.

(12) Slotine, J.J. and Sastry, S.S., Tracking Control of Nonlinear Systems Using Sliding Surfaces, with Application to Robot Manipulations, Int. J. Control, Vol. 38, No. 2 (1983), p. 465.

(13) Utkin, V.I., Discontinuous Control System: State of Art in Theory and Applications, Preprint 10th IFAC World Congr., Vol. 1 (1987), p. 75.

(14) Hikita, H., Servomechanisms Based on Sliding Mode Control, Int. J. Control, Vol. 48, No. 2 (1988), p. 435.

(15) Hikita, H., Kubo, H. and Narita, T., Design of Servomechanisms Using Sliding Mode and QuasiSliding Mode, Trans. Instit. Syst. Contr. and Inform. Eng., (in Japanese), Vol. 1, No. 2 (1988), p. 53. 